\title{
Globe
}

Revue internationale d'études québécoises

\section{Vice et Versa, dix ans après}

\section{Gilles Dupuis}

Volume 13, numéro 2, 2010

URI : https://id.erudit.org/iderudit/1001136ar

DOI : https://doi.org/10.7202/1001136ar

Aller au sommaire du numéro

\section{Éditeur(s)}

Globe, Revue internationale d'études québécoises

\section{ISSN}

1481-5869 (imprimé)

1923-8231 (numérique)

Découvrir la revue

Citer cette note

Dupuis, G. (2010). Vice et Versa, dix ans après. Globe, 13(2), 187-194.

https://doi.org/10.7202/1001136ar d'utilisation que vous pouvez consulter en ligne.

https://apropos.erudit.org/fr/usagers/politique-dutilisation/ 


\section{NOTE CRITIQUE}
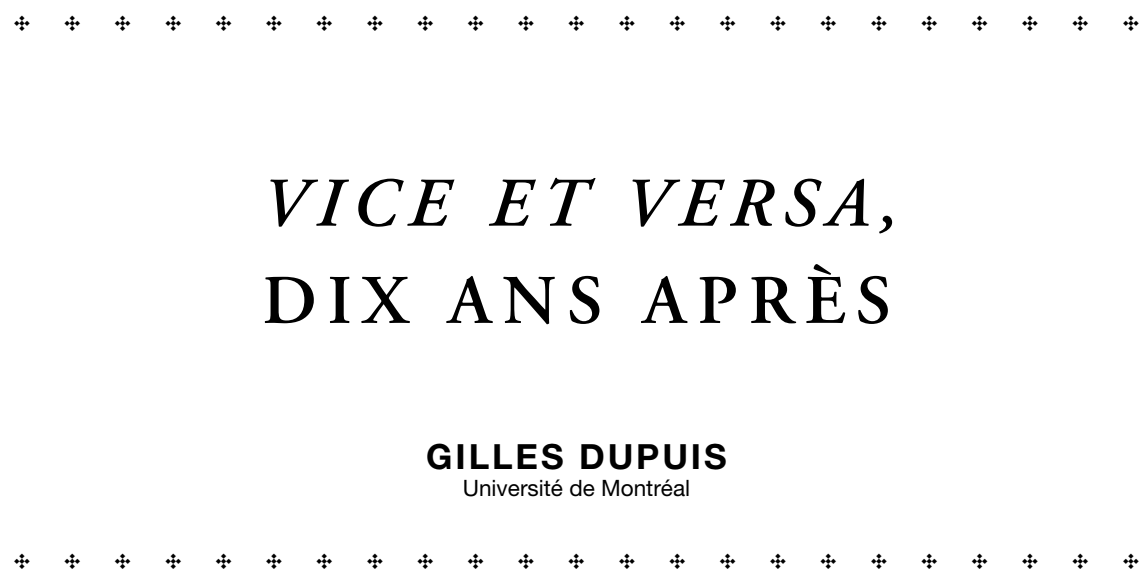

Publiés à quatre ans d'intervalle, à partir de colloques qui se sont déroulés à deux ans de distance, les deux ouvrages collectifs, Le projet transculturel de "Vice Versa ${ }^{1}$ " et La transculture et Vice Versa ${ }^{2}$, reviennent sur l'expérience stimulante et mouvementée des quatorze années d'existence (1983-1997) de l'unique revue transculturelle du Québec afin d'en proposer un bilan critique, voire autocritique, à partir de perspectives complémentaires qui se recoupent autour de voix communes. Mon propos dans cette note critique n'est pas de rendre compte in extenso de la richesse de ces deux bilans qui venaient à point nommé pour marquer et même anticiper la commémoration du dixième anniversaire de la disparition intempestive de la revue, mais d'en faire ressortir les lignes de force et les «lieux » où ces lignes se sont rencontrées.

$$
\div \div
$$

1. Le projet transculturel de "Vice Versa", Actes du Séminaire international du CISQ à Rome (25 novembre 2005), sous la direction d'Anna Paola MosseTTO, Bologne, Pendragon, 2006. Les références à ce volume seront indiquées dans le texte entre parenthèses.

2. La transculture et Vice Versa, sous la direction de Fulvio CACCIA, Montréal, Triptyque, 2010. Les références à ce volume seront indiquées dans le texte entre parenthèses. 


\section{VICE VERSA VU DE LITALIE}

En organisant un colloque, aussi modeste fût-il quant au nombre des participants, autour du projet transculturel de Vice Versa en 2005, le Centre interuniversitaire d'études québécoises en Italie (CISQ) faisait d'une pierre deux coups : il anticipait de deux ans la commémoration qui allait sûrement venir de l'autre côté de l'Atlantique, tout en invitant le « rejeton " au berceau qui avait vu naître les fondateurs de la revue. C'est donc en Italie et non au Québec, lieu de naissance officiel de Vice Versa, qu'un premier bilan critique $\mathrm{du}$ "projet transculturel » de la revue a vu le jour. Et c'est à Rome, non à Bologne ou à Turin comme on aurait pu s'y attendre, que le CISQ a choisi de faire le point sur les quatorze ans d'existence du magazine. Le choix de la «ville ouverte» (du nom qu'avait donné le cinéaste Roberto Rossellini à la Ville éternelle) était en soi une gageure en faveur de la transculture dont le projet (en particulier en Europe) reste à venir. Ce premier bilan a été effectué, entre autres, par deux membres fondateurs de Vice Versa, Lamberto Tassinari et Fulvio Caccia, qui ont souvent agi comme porte-parole intellectuels de la revue, par un membre un peu plus tardif de son comité de rédaction, Régine Robin, qui y a parfois joué le rôle de critique interne, et par un sympathisant à sa cause, Pierre Nepveu.

D'entrée de jeu, l'ex-directeur de la revue, le philosophe Tassinari, rappelle qu'il n'y a jamais eu à Vice Versa de consensus autour de la notion de transculture : «chacun aura donné sa version personnelle, sa propre interprétation de la transculture, car une formulation officielle, définitive, n'a jamais été livrée durant les années d'activité du magazine». (p. 17) L'idée même d'un consensus à atteindre, a fortiori quand il s'agit de débattre d'un concept aussi complexe (et, il faut bien le dire, controversé) que celui de transculture, était étrangère à l'esprit qui animait l'équipe de la revue. Néanmoins, ses animateurs voulaient "donner une forme théorique mais aussi $[. .$.$] pratique"$ (p. 19) à leur projet transculturel, dans le contexte global de la mondialisation et de l'immigration généralisée, en assumant à la fois leur position marginale et le caractère utopique, mais nécessaire, de leur engagement intellectuel au sein de la polis. Pour Tassinari, et d'autres qui l'ont suivi dans le même sens, le «trans [...] proposé par "Vice Versa", signifiait traversée, passage, métamorphose continue de l'identité : perte et gain sans arrêt, osmose ". (p. 23) Le sujet transculturel conservait des éléments de son identité d'origine, en abandonnait d'autres, tout en acquérant de nouveaux traits culturels par une forme de contamination salutaire avec les diverses cultures qu'il traversait (ou mieux, qui le traversaient). La transculture se projetait ainsi d'aller beaucoup plus loin que le simple contact interculturel, ce qui explique sans 
doute son caractère utopique sur le plan politique, le Québec ayant opté, à la suite des travaux de la Commission Bouchard-Taylor, pour le modèle interculturel qui lui était antérieur ${ }^{3}$.

Se réclamant de Marc Fumaroli et de Robert Richard, Fulvio Caccia situe le projet transculturel de Vice Versa au fondement d'une "république des lettres" (p. 31). Fidèle à son propre projet idéologique de fondation d'une "république $M e ̂ t i s^{4}$ ", qui aurait introduit dans le modèle républicain français l'élément qui lui manquait du métissage - projet d'ailleurs repris plus timidement par Pascale Casanova ${ }^{5}-$, Caccia définit ainsi le défi posé à toute république transculturelle : « fonder un corps politique en dehors de toute communauté d'esprit, de langue et de culture». (p. 33) Plus radical que ses confrères italiens à Vice Versa (Lamberto Tassinari, Bruno Ramirez et surtout Antonio D’Alfonso), il se rapproche de la position défendue par Régine Robin lorsqu'elle refuse de jouer à la revue « la juive de service» (p. 76), et par extension le rôle de représentante d'une quelconque communauté ethnique ou culturelle, au nom d'« un vrai métissage culturel, une imprégnation des cultures, un processus de passage, de mouvement, de métamorphose». (p. 77) Consciente des ratés du magazine, qu'elle impute en grande partie, à tort ou à raison (j'opterais pour «à raison et à tort»), au nationalisme québécois et à la fin du financement de la part des instances provinciale et fédérale, elle propose une formule qui me paraît néanmoins irréfutable : l'échec de Vice Versa fut "un échec productif». Productif en ce sens où le magazine aura été au Québec, mais aussi à l'extérieur de ses frontières, "un vrai laboratoire de la postmodernité, de la transculture ". (p. 77)

Parmi les invités aux agapes romaines, Pierre Nepveu était le seul Québécois issu de la majorité francophone d'origine canadienne-française. Sympathique au projet de Vice Versa, comme son collègue trop tôt disparu, Pierre L'Hérault, il abonde dans le sens de Régine Robin en rappelant que dans ses grandes années, qu'il situe entre 1985 et 1989, «la majeure partie de l'intelligentsia québécoise est demeurée à distance de la revue, y voyant surtout un curieux phénomène, un étrange objet dont on ne savait trop quoi faire». (p. 83) Il note le peu d'intérêt que des revues intellectuelles comme

$$
4+4
$$

3. Voir à ce sujet l'essai de Gérard BOUCHARD, "Laïcité. La voie québécoise de l'interculturalisme", Jean-François Plamondon et Anne de Vaucher (dir.), Les enjeux du pluralisme. L'actualité du modèle québécois, Bologne, Pendragon, 2010, p. 109-142.

4. Fulvio CACCIA, La république Mêtis, Montréal, Balzac-Le Griot, 1997.

5. Pascale CASANOVA, La République mondiale des lettres, Paris, Seuil, 1999. 
Liberté et Possibles ont manifesté à l'endroit des concepts avancés par Vice Versa, même s'il reconnaît, d'un même souffle, que celui de "transculture", sur lequel il a lui-même réfléchi ${ }^{6}$, "était plus une pratique qu'un concept, relevait davantage du performatif que de l'analyse». (p. 84) Il va pourtant plus loin que les fondateurs de la revue lorsqu'il affirme, à contre-courant de l'intelligentsia nationaliste de l'époque et celle qui viendra par la suite, que la pensée transculturelle à Vice Versa "apparaît de ce point de vue comme une position d'avant-garde, peut-être comme la dernière vraie avant-garde qui se soit manifestée au Québec». (p. 90) Il aurait été difficile de la part de cette élite intellectuelle, pour les raisons que l'on devine aisément même si on ne les partage pas, d'admettre que la "dernière vraie avant-garde » ait été largement le fait d'immigrants plutôt que de bons vieux Québécois de souche... Un accueil semblable sera réservé aux " écritures migrantes ", élaborées dans le creuset de Vice Versa, avant qu'elles ne soient récupérées par l'institution littéraire québécoise.

Les Actes du colloque de Rome sont complétés par deux interventions venant de la part de chercheurs italiens (Claudio Strinati et Franco Ferrarotti), qui s'emploient avec plus ou moins de bonheur à trouver un pendant au concept de "transculture " en Italie, et par une contribution majeure de Gianni Caccia, le directeur artistique de Vice Versa de 1983 à 1993, lequel retrace les débuts difficiles et la suite gratifiante de l'aventure graphique de la revue tout en rendant hommage aux collaborateurs de l'heure, dont le regretté Vittorio. Dans sa préface au volume, Anna Paola Mossetto, alors directrice du CISQ, rappelait le caractère à la fois utopique et courageux de la revue et de ses artisans, en insistant sur la dimension «épique " de son projet transculturel. Belle façon de saluer l'épopée inachevée de la revue, à une époque où plus que jamais on aurait besoin d'une «bonne trans-culture » (Tassinari, p. 19).

\section{VICE VERSA REVU D'ICI}

C'est au Québec qu'a eu lieu la commémoration «officielle» de Vice Versa, et à ma connaissance la seule qui ait coïncidé avec les dix ans de la disparition de la revue. Ici aussi une précision s'impose : c'est bien à Montréal, comme il se devait, que le deuxième bilan critique du projet transculturel de la revue a vu le jour ; or c'est à l'Université Concordia, et non à la prestigieuse université anglophone de la métropole ou dans l'une de ses deux consœurs

$$
\div+\div
$$

6. Pierre NePveu, «Qu'est-ce que la transculture?», Paragraphes, n 2, 1989, p. 15-31. 
francophones, que l'événement s'est déroulé. Question de stratégie ou d'ouverture?

Quoi qu'il en soit, les organisateurs avaient fait appel pour l'occasion à une vingtaine de participants qui sont intervenus pendant les trois jours que durait la rencontre. Outre les membres fondateurs issus de l'intelligentsia italo-québécoise et les concepteurs graphiques de la revue, le colloque de Montréal intitulé "Diversité culturelle et transculture ou Vice Versa. Qu'est-ce que la transculture aujourd'hui ? " a réuni bon nombre d'intellectuels, de personnalités et d'écrivains qui avaient tous en commun d'avoir fait partie à un moment ou un autre du comité de rédaction de Vice Versa, d'avoir collaboré ponctuellement à la revue, ou de s'être intéressé de près ou de loin à son projet transculturel. Le riche bilan qui en découle (seize contributions individuelles plus une discussion réunissant quatre participants), représentatif des différents secteurs du milieu culturel québécois qui ont animé l'aventure intellectuelle et créatrice du magazine, complète, tout en l'élargissant, le premier bilan réalisé en Italie.

Il m'est impossible, dans les pages qui me sont imparties, de rendre compte équitablement de toutes les contributions qui sont venues enrichir cette deuxième mise au point de l'aventure transculturelle de Vice Versa. Par conséquent, je vais m’en tenir à ce qui m’apparaît le plus essentiel pour une mise en perspective de la fortune critique du magazine, soit les témoignages théoriques regroupés sous l'intitulé "Transcultures». Avant d'entrer dans les détails, je tiens cependant à faire remarquer que le volume s'ouvre par un poème "transculturel " du géographe métis Jean Morisset, glosé par l'auteur, et se clôt sur un conte de l'écrivaine d'origine marocaine, Myriame El Yamani, illustrant à la fois la place importante qu'occupait la création dans la revue et le rôle de premier plan joué par les auteurs «migrants » ou "hybrides », d'origines multiples et croisées, dans le développement de son concept de transculture. Les témoignages plus personnels d'Évelyne Abitbol, de Karim Moutarrif et de Robert Berrouët-Oriol frayent dans le même sens.

Je n'insisterai pas davantage sur les contributions des membres fondateurs du magazine, qui racontent les débuts incertains mais excitants de la revue (Bruno Ramirez et Antonio D’Alfonso), les défis stimulants posés par la maquette et les illustrations (Gianni Caccia et ses confrères), ou qui reviennent sur les idées déjà avancées dans le bilan romain (Lamberto Tassinari et Fulvio Caccia), sinon pour rappeler « la nature implicitement politique [du] projet» (p. 131) de Vice Versa, que Tassinari lie non plus à la postmodernité québécoise mais au postcolonialisme canadien, et "la 
transfiguration de la condition humaine " (p. 195) évoquée par Caccia lorsqu'il rapproche le transculturel du trasumanar chez Dante. Les autres contributions théoriques du collectif oscillent entre trois perspectives que je qualifierais d'archéologique, d'actuelle et de futuriste: tenter une généalogie du concept de transculturation avant l'expérience vice-versienne (Walter Moser), faire le point sur l'apport de Vice Versa à la théorisation du phénomène transculturel (Christian Roy, Nicolas van Schendel, Pierre Ouellet et Simon Harel) et proposer de nouvelles pistes de réflexion après le deuil de la transculture (William Anselmi).

Commençons par l'actualité du phénomène. Dans «L'empire des périphéries: le Canada, Mitteleuropa du Nouveau Monde ", Christian Roy élargit l'expérience québécoise de Vice Versa au cas du Canada dans son entier, élargissement qui avait d'ailleurs été entrepris par le comité de direction du magazine dans les dernières années de sa vie. Le rapprochement effectué par Roy entre le Canada et la Mitteleuropa lui permet d'avancer des propos paradoxaux, sans doute contestables mais fort intéressants, comme le fait que le Canada serait "un Empire multinational de facto" (p. 67) et que «la persistance d'une monarchie en Amérique pourrait bien être vue comme un symbole par excellence de la nature transculturelle du projet canadien au degré zéro de sa singularité, si l'on entend par transculture "la traversée sans l'oubli" (Tassinari)» (p. 69). De tels propos, qui vont diamétralement à l'encontre de la conception républicaine que se fait un Fulvio Caccia d'une "politique transculturelle", montrent en quoi le terme peut signifier autrement dans un contexte plus large que celui où il a été pensé originairement. Dans une même optique canadienne et «transnationale», Nicolas van Schendel («Identités américaines et potentialités transculturelles : vers une nouvelle canadianité ?») revient sur la notion de voix «métisses " proposée naguère par Robert Berrouët-Oriol ${ }^{7}$, mais pour l'infléchir dans le sens d'une identité politique. Il oppose ainsi «l'Être mosaïque " à la "figure du Métis», le premier étant, si je simplifie, «interculturel» alors que la seconde serait "transculturelle». En valorisant la figure du métis, van Schendel se rapproche de Caccia tout en intégrant à sa réflexion l'Autre "autochtone», somme toute peu présent dans l'aventure vice-versienne.

Pour sa part, Pierre Ouellet insiste sur « la structure hétérotopique " de Vice Versa en tant que "reconfiguration à la fois territoriale et culturelle de la citoyenneté ou de la coexistence au sein de la Cité». (p. 109)

$\div+\div$

7. Robert BERROUËT-OrIOL, «L'effet d'exil », Vice Versa, nº 17, décembre 1986/janvier 1987, p. 20-21. 
Tout en restreignant l'engagement politique de la revue à la ville (Montréal, Toronto, New York, peu importe), il lui donne une dimension universelle que les conceptions "nationalistes" (québécoise ou canadienne), pourtant plus vastes géographiquement, n'arrivent pas à englober. Enfin, Simon Harel rejoint Pierre Nepveu quand il prétend que Vice Versa a incarné « une véritable avant-garde dans le domaine des lettres québécoises" (p. 116), à un moment crucial où le Québec ne parvenait pas à se dégager de l'impasse du premier référendum. En insistant sur la dimension «subversive» (p. 118) de la pensée transculturelle, il conclut son texte en rappelant que "les écrivains italo-québécois de la revue Vice Versa auront sans doute été les premiers à faire le deuil de leur ethnicité afin de promouvoir une nouvelle forme de représentation du collectif québécois». (p. 128) Le mot "deuil» me semble ici excessif, eu égard notamment aux aveux ethnicisants d'un D'Alfonso, voire de Tassinari lui-même quand il parle de "traversée sans oubli»; en revanche, l'ouverture à de nouvelles façons d'être collectivement québécois ou, à tout le moins, de penser autrement ce collectif peut être attribuée à un renoncement (partiel ou complet) au discours des origines.

En amont et en aval du bilan intensif et extensif proposé par tous ces intervenants au colloque se trouvent deux autres contributions de longueur inégale, certes, mais également pertinentes dans l'appréhension des bornes temporelles du phénomène transculturel théorisé à Vice Versa: une contribution majeure de Walter Moser intitulée "Transculturation : métamorphoses d'un concept migrateur", et l'essai provocant de William Anselmi, «Du "transitionnisme" ".

Dans son article de fond, Moser retrace la genèse du mot «transculture" au concept de transculturación avancé par l'anthropologue cubain Fernando Ortiz afin de rendre compte du type particulier de métissage, impliquant un double processus de déculturation et de néoculturation, dont son île natale aurait été le théâtre à la suite de sa colonisation par l'Espagne suivie de sa phase de décolonisation opérée par l'État. Transplantée par les Vice-versiens dans le contexte québécois, après «sa généralisation latinoaméricaine " (à travers la notion d'anthropophagie culturelle au Brésil par exemple), "la transculturalité " y fut mal accueillie car elle "pouvait être perçue comme une provocation, si ce n'est une agression contre l'identité québécoise ", reposant elle-même sur les «efforts de faire du français l'unique langue officielle du Québec." (p. 48-49) Sur ce point l'analyse de Moser rejoint le constat de Nepveu, bien qu'il s'emploiera par la suite à critiquer la réduction ontologique qu'opère ce dernier du concept de transculturation «en faisant une petite erreur de lecture symptomatique» (p. 53) d'un passage 
de Heidegger qu'il attribue au «langage " alors qu'il concerne un «entretien » sur le langage. Je ne peux adéquatement rendre compte ici de l'analyse subtile que fait Moser du glissement de lecture commis par Nepveu. Ce qu'il faut retenir de sa propre lecture critique par contre, c'est la nécessité de conserver au concept de transculture sa valeur "épistémique» (p. 57) que sa réduction ontologique (ou pire, sa récupération institutionnelle) lui avait retirée.

Il revient à William Anselmi, le seul représentant du Canada anglophone (professeur à l'Université d'Alberta) au colloque et possiblement le seul intervenant à n'avoir pas été associé directement à Vice Versa, de proposer une sortie hors de la sphère transculturelle et de la postmodernité qui lui était associée, en argumentant en faveur d'un "transhumanisme» (p. 92) - sans doute pas très éloigné du trasumanar dantesque théorisé par Fulvio Caccia - et pour une forme de «transitionnisme » (p. 99) dont le projet, forcément inachevé, devra demeurer ouvert. Mais alors, qu'est-ce qui distingue, sur le plan épistémologique, voire heuristique, «un transculturalisme compassionnel » (qui serait la version accueillante ou mieux, recueillante de la transculture selon Nepveu) de «l'évolution de l'humanisme» (qui en constituerait la version ouverte, jamais achevée d'après Moser) ? Au seuil de cette énigme, qui n'est peut-être que la formulation maladroite d'un faux dilemme, je préfere laisser la question en suspens. 